\title{
Results from the Double Chooz experiment
}

\author{
Michiru Kaneda*† \\ Tokyo Institute of Technology \\ E-mail: kanedadhep.phys.titech.ac.jp
}

The Double Chooz experiment is a neutrino experiment using electron antineutrinos produced by reactor cores of the Chooz nuclear power plant. The main purpose of the experiment is to measure the neutrino mixing angle $\theta_{13}$. Non-zero mixing angle results in a disappearance of electron antineutrinos. The experiment consists of two detectors. The far one is located at a distance of $1050 \mathrm{~m}$ from the reactor cores to measure the disappearance of electron antineutrinos, while the near one is located at a distance of $400 \mathrm{~m}$ from the reactor cores to measure the original neutrino flux from the reactor cores. The data taking started in 2011 at the far detector, and in 2014 at the near detector. In this presentation, the latest results from the Double Chooz experiment are shown. The main result of the neutrino mixing angle $\theta_{13}$ value is $\sin ^{2} 2 \theta_{13}=0.119 \pm 0.016$.

XIV International Conference on Heavy Quarks and Leptons (HQL2018)

May 27- June 1, 2018

Yamagata Terrsa, Yamagata,Japan

\footnotetext{
*Speaker.

${ }^{\dagger}$ On behalf of the Double Chooz Collaboration
} 


\section{Introduction}

Neutrino oscillations are parameterized by the three mixing angles $\left(\theta_{12}, \theta_{13}, \theta_{23}\right)$, three mass squared differences $\left(\Delta m_{21}^{2}, \Delta m_{31}^{2}, \Delta m_{32}^{2}\right)$ and a CP-violating phase $\left(\delta_{C P}\right)$. They have been measured in solar, atmospheric, accelerator and reactor experiments[四]. Main purpose of reactor neutrino

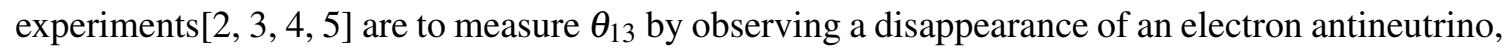
$\bar{v}_{e}$. The energy of $\bar{v}_{e}$ from reactor cores is about a few $\mathrm{MeV}$. The remaining probability of $\bar{v}_{e}$ of a few $\mathrm{MeV}$ up to a few $\mathrm{km}$ from the rector cores can be written as follows:

$$
P_{\bar{v}_{e} \rightarrow \bar{v}_{e}}=1-\sin ^{2} 2 \theta_{13} \sin ^{2}\left(\frac{1.27 \Delta m_{13}^{2} L}{E_{\bar{v}_{e}}}\right),
$$

where $L$ is a distance and $E_{\bar{v}_{e}}$ is energy of $\bar{v}_{e}$. By using $\Delta m_{13}^{2}$ measured by the MINOS experiment, $\Delta m_{13}^{2}=2.44_{-0.10}^{+0.90}[$ [ $]$, the first maximum oscillation point is expected around $1 \mathrm{~km}$.

To detect $\bar{v}_{e}$, experiments use the inverse $\beta$-decay (IBD) process $\left(\bar{v}_{e}+p \rightarrow e^{+}+n\right)$ in liquid scintillator detectors. In IBD process, a positron and a neutron are emitted and they produce two time-different events, prompt and delayed events, respectively. The positron immediately annihilates with an electron and emits gamma rays. These gamma rays are caught by the detector and the total energy $E$ has a relation to the incoming neutrino energy as follows:

$$
E \simeq E_{v}-0.8 \mathrm{MeV}
$$

where $E_{v}$ is the energy of the incoming neutrino. The neutron is thermalized and captured by a nucleus. To enhance the neutron event, usually, gadolinium (Gd) doped liquid scintillator is used. The Gd capture event occurs in a mean delay time of $30 \mu \mathrm{s}$ after the positron event, and emits photons with a total energy of $8 \mathrm{MeV}$. The neutron is captured by hydrogen $(\mathrm{H})$, too. The $\mathrm{H}$ capture event's mean delay time is $200 \mu$ s and the total energy of emitted photons is $2.2 \mathrm{MeV}$. The $\mathrm{Gd}$ capture event is easier to be separated from backgrounds as higher energy and short delay time, while the $\mathrm{H}$ capture event has more statistics. IBD events can be selected by using two continuous events' correlations. Background events can be strongly suppressed by the coincidence of these prompt and delayed signals.

\section{The Double Chooz experiment}

The Double Chooz experiment uses reactor cores of the Chooz nuclear power plant in France. There are two reactor cores of $4.25 \mathrm{GW}_{\text {th }}$ power. Two reactors are placed within $170 \mathrm{~m}$. Therefore, the neutrino flux from these reactor cores is almost iso-flux. In addition, there are some chances to take both reactor cores off data as there are only two reactor cores.

The experiment consists of two detectors, the Far Detector (FD) and the Near Detector (ND), located at distances of $1050 \mathrm{~m}$ and $400 \mathrm{~m}$ from the reactor cores, respectively. FD is placed around the first maximum oscillation point to measure the disappearance of $\bar{v}_{e}$, while ND measures original $\bar{v}_{e}$ flux from reactor cores for the comparison. They are placed underground of $300 \mathrm{~m}$. w. e. depth and $120 \mathrm{~m}$. w. e. depth, respectively. These two detectors' designs are identical. Detectors consist of the Inner Detector (ID), the Inner Veto (IV) and the Outer Veto (OV). ID is the main detector to detect $\bar{v}_{e}$. The innermost part of ID is filled with $10 \mathrm{~m}^{3} \mathrm{Gd}$ doped liquid scintillator and called 
the Neutrino Target (NT). Around NT, there is $55 \mathrm{~cm}$ thick Gd-free liquid scintillator region, called the Gamma Catcher (GC), to capture escaped photons from NT. H capture events can occur in both NT and GC. while the Gd capture events occur only in NT. GC is surrounded by the Buffer region filled with mineral oil, in which 390 photomultiplier tubes are set. Separated by the stainless steel vessel, IV surrounds ID to identify background events related to radiation sources from outside of the detector. IV is $55 \mathrm{~cm}$ thick liquid scintillator layer and 78 photomultiplier tubes are set in there. OV is a plastic scintillator detector covers the top of the detector. It is used to identify cosmic muon background events.

The data taking started in 2011 with only FD, and ND started data taking in 2014.

\section{Dataset}

The dataset is separated into three, FD-I, FD-II, and ND, by the detectors and the period. FD-I is FD data taken before ND started, while FD-II is data of FD running with ND. In FD-I data, there are about 7 days reactor cores off data.

\section{Event selection}

Events are rejected if they are within $1.25 \mathrm{~ms}$ after a muon event or identified as a light noise event. IBD event candidates are selected if two continuous events (prompt and delayed) satisfy conditions of the prompt energy, the delayed energy, the time difference of two events and the distance of two events as: $1 \mathrm{MeV}<E_{\text {prompt }}<20 \mathrm{MeV}, 1.3 \mathrm{MeV}<E_{\text {delayed }}<10 \mathrm{MeV}, 0.5 \mu \mathrm{s}<\Delta T<$ $800 \mu \mathrm{s}$ and $\Delta R<1200 \mathrm{~mm}$, respectively. If there is another event before $800 \mu \mathrm{s}$ and after $900 \mu \mathrm{s}$ of the prompt event, the candidate is rejected. Events with OV hits are rejected as muon events. IV information is used to veto fast neutrons and accidental events. Vertex reconstruction goodness is used to remove stopping muon events. A likelihood is constructed from the prompt energy and the time difference, to reject cosmogenic background events. An event selection using Artificial Neural Network (ANN) based on $E_{\text {delayed }}, \Delta T$ and $\Delta R$ is used to suppress accidental events.

\section{Result}

$\theta_{13}$ is measured by a simultaneous fit of data and prediction on the event rate and the prompt energy in each dataset. The prediction is constructed with signal Monte Carlo and backgrounds estimated from data. Reactor off data is used to constrain backgrounds. Systematic uncertainty correlations between datasets are considered in the fit. Figure $\mathbb{W}$ shows the ratio of data to the no oscillation prediction for FD-I, FD-II and ND. The best fit value is $\sin ^{2} 2 \theta_{13}=0.119 \pm 0.016$ and $\chi^{2} / \mathrm{ndf}$ is $236.2 / 114$.

Another fit is performed using same period data of FD-II and ND for the cross-check. Figure $\square$ shows the ratio of FD-II to ND and the best fit value is $\sin ^{2} 2 \theta_{13}=0.123 \pm 0.023 . \chi^{2} /$ ndf is $10.6 / 38$.

\section{Prospect}

The experiment continued data taking until early 2018 and the total data will be doubled. The new data contain a few tens days reactor off data and it is new for ND. 
Systematic uncertainty evolution of Double Chooz is shown in Figure [3. The reactor flux was the dominant uncertainty before ND data was included, while it is strongly suppressed by ND data in the recent results. Current largest systematic uncertainty is the detection uncertainty, which is dominated by the proton number uncertainty in ID. This uncertainty can be suppressed by the direct measurement at the deconstruction of the detectors.

Figure 4 shows sensitivity prospect of the Double Chooz experiment. By improving the proton number uncertainty, the total uncertainty less than 0.01 can be achieved.

In addition, there are some analyses other than $\theta_{13}$, such a sterile neutrino search and neutrino directionality. These results also will be available soon.

\section{Conclusion}

The Double Chooz results of $\theta_{13}$ using two-detector system are presented. The data to prediction simultaneous fit results $\sin ^{2} 2 \theta_{13}=0.119 \pm 0.016$. Current dominant uncertainty is the proton number uncertainty in ID. This can be suppressed by the direct measurement at the deconstruction of the detectors, and the total uncertainty less than 0.01 is expected.

\section{References}

[1] M. Tanabashi et al. (Particle Data Group), Phys. Rev. D 98, 030001 (2018).

[2] Y. Abe et al., JHEP 10, 086 (2014).

[3] Y. Abe et al., JHEP 01, 163 (2016).

[4] F.P. An et al., Phys. Rev. Lett. 116, 061801 (2016).

[5] J.H. Choi et al., Phys. Rev. Lett. 116, 211801 (2016).

[6] P. Adamson et al., Phys. Rev. Lett. 112, 191801 (2014). 

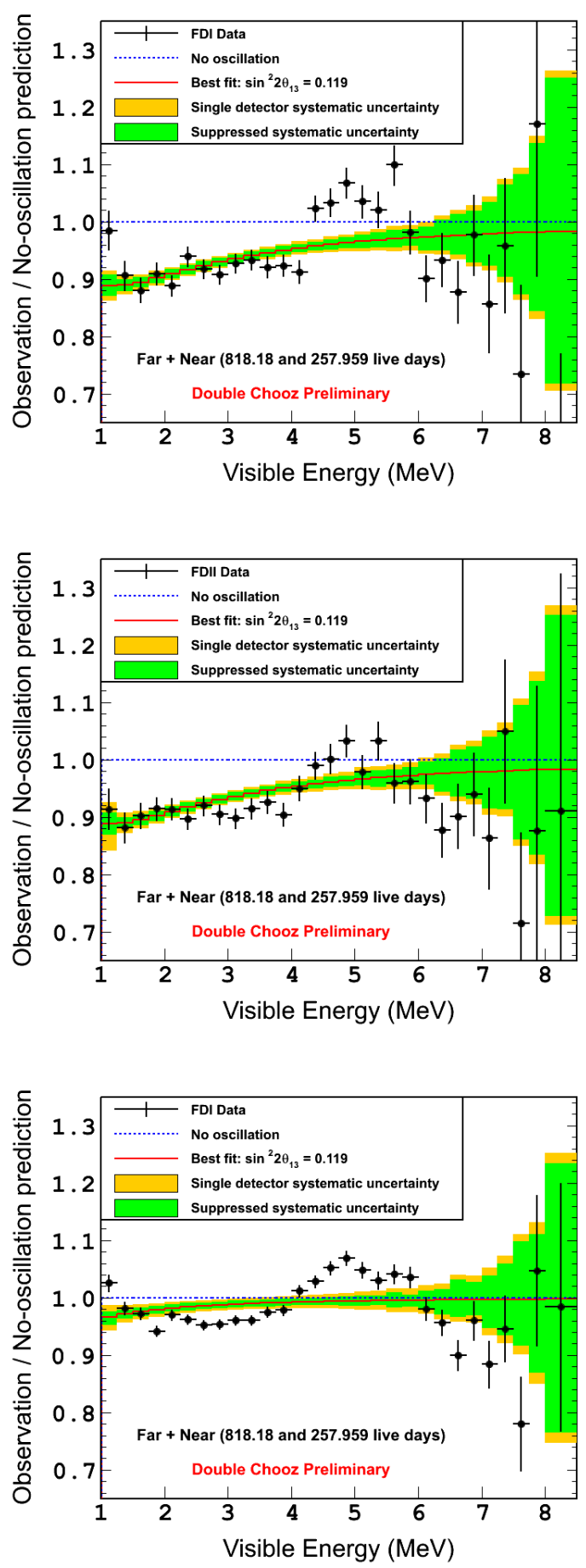

Figure 1: The prompt energy ratio of data to the no oscillation prediction for FD-I, FD-II and ND. 


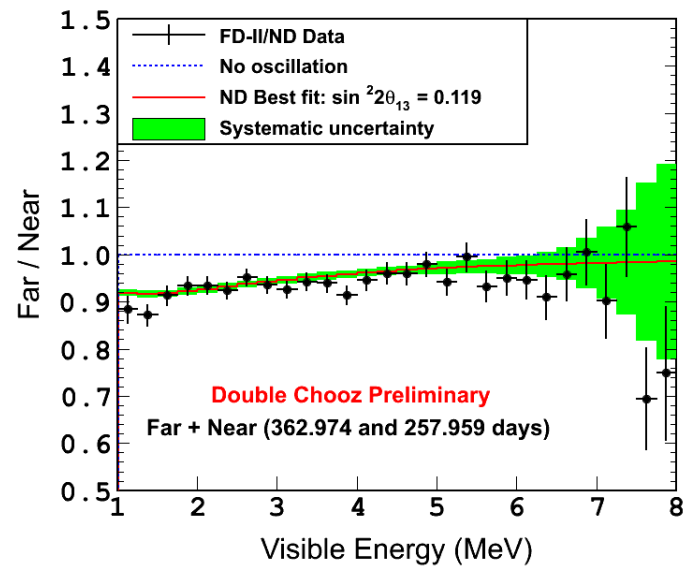

Figure 2: The prompt energy ratio of FD-II to ND.

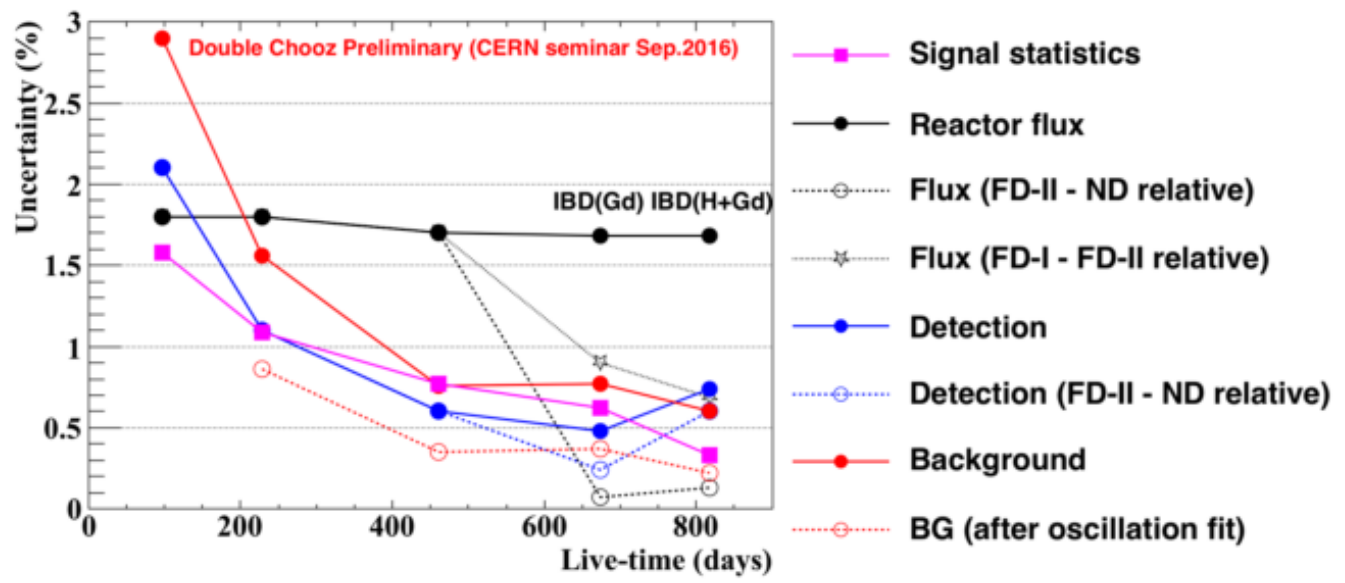

Figure 3: Systematic uncertainty evolution. 


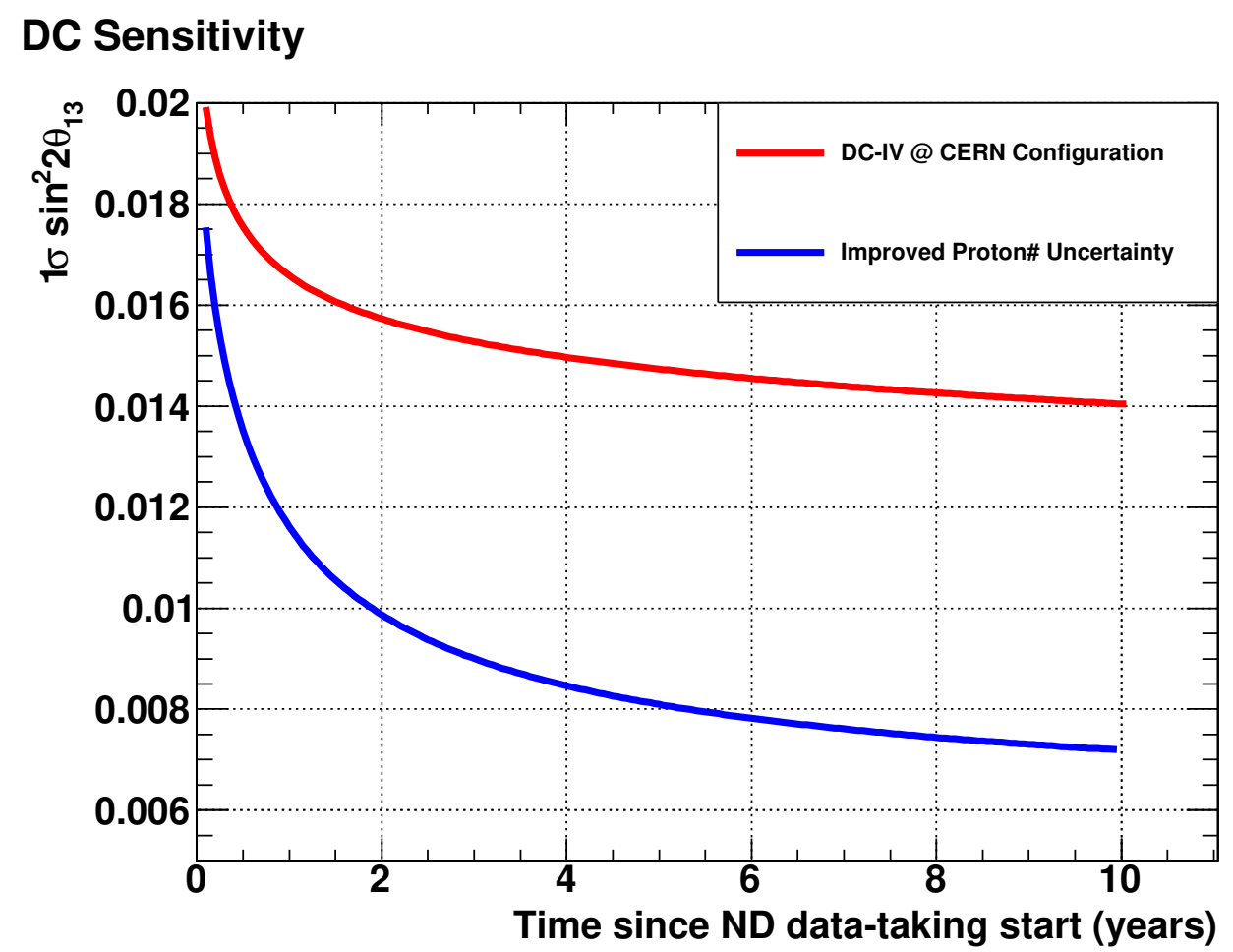

Figure 4: Double Chooz sensitivity prospect. 